\title{
Pneumocystis Pneumonia and Acute Myeloid Leukemia: A Systematic Review
}

\author{
Atousa Hakamifard ${ }^{1}$, Shervin Shokouhi ${ }^{1}$, Masoud Mardani ${ }^{1}$, Nariman Mosaffa ${ }^{1}$, and \\ Hamed Azhdari Tehrani ${ }^{1}$ \\ ${ }^{1}$ Shahid Beheshti University of Medical Sciences
}

July 15, 2021

\begin{abstract}
Abstract: Background: Pneumocystis pneumonia (PCP) incidence in acute myeloid leukemia (AML) patients have not been well described and some studies have shown the risk of this infection in these groups of patients. In this systematic review, we reviewed the published studies about PCP in AML to evaluate the PCP incidence and outcomes in these patients and then focuses on its immunological mechanism. Method: All articles reviewed in this collection are from recruited sites: PubMed and Embase databases. No time limit was considered for article searching. The following keywords were used: ("Pneumonia, Pneumocystis" OR Pneumocystis Pneumonia" OR "Pneumocystis jirovecii"), AND ("leukemia, myeloid, acute OR acute myeloid leukemia"). Our search strategy yielded 356 articles. After implementing the exclusion and inclusion criteria, the final papers were selected and reviewed. Result: By our search, 356 articles were found (316 in PubMed and 40 in Embase). After the implementation of inclusion and exclusion criteria, seven papers remained. A total of seven articles with a total number of 41 patients were included. Conclusion: Affected patients appear to have a clear resistance to PCP infection despite chemotherapy and bone marrow suppression due to the preservation of a specific immunological milieu in the lung. In fact, may be due to immunological stability of the lungs that remains intact. Further studies are needed.
\end{abstract}

\section{Pneumocystis Pneumonia and Acute Myeloid Leukemia: A Systematic Review}

\section{Abstract:}

Background: Pneumocystis pneumonia (PCP) incidence in acute myeloid leukemia (AML) patients have not been well described and some studies have shown the risk of this infection in these groups of patients. In this systematic review, we reviewed the published studies about PCP in AML to evaluate the PCP incidence and outcomes in these patients and then focuses on its immunological mechanism.

Method: All articles reviewed in this collection are from recruited sites: PubMed and Embase databases. No time limit was considered for article searching. The following keywords were used: ("Pneumonia, Pneumocystis" OR Pneumocystis Pneumonia" OR "Pneumocystis jirovecii"), AND ("leukemia, myeloid, acute OR acute myeloid leukemia"). Our search strategy yielded 356 articles. After implementing the exclusion and inclusion criteria, the final papers were selected and reviewed.

Result: By our search, 356 articles were found (316 in PubMed and 40 in Embase). After the implementation of inclusion and exclusion criteria, seven papers remained. A total of seven articles with a total number of 41 patients were included.

Conclusion : Affected patients appear to have a clear resistance to PCP infection despite chemotherapy and bone marrow suppression due to the preservation of a specific immunological milieu in the lung. In fact, may be due to immunological stability of the lungs that remains intact. Further studies are needed.

Review criteria: how did you gather, select and analyze the information you considered in your review? 
We have searched all of the databases and literature associated with this topic in Pubmed, Google Scholar, EMBASE databases. Then, We proceed regard to PRISMA flowchart. In this review, there have been identified 356 articles.

Message for the clinic: what is the 'take-home' message for the clinician?

the aim of this study was to evaluate what is the probability of PCP infection and acute myeloid leukemia and then evaluate why this probability is low in this certain population. So this is always an important question for us that how the difference between myeloid leukemia and lymphoid leukemia would be justified.

Keywords: Acute myeloid leukemia, pneumocystis pneumonia, pneumonia, pneumocystis jirovecii, pneumocystis, systematic review

\section{Pneumocystis pneumonia and acute myeloid leukemia: a systematic review}

\section{Introduction}

There are several important issues about patients with immunosuppression especially patients with malignancy. One of the most important of them is the higher risk of opportunistic infections which are common among them especially in patients with hematologic malignancies, who have received intensified chemotherapeutic regimens $[1,2]$. As we see in literature there are many aspects that may be questionable during routine clinical practice and some of them would change according to new findings in clinical guidelines. The major aim of prescribing prophylactic antimicrobial therapy is to reduce the infection related morbidity and mortality [3] and the overwhelming possible infections related complications which may reduce the treatment tolerability, so use of prophylactic antimicrobials were introduced from long time ago and that was widely studied $[4,5]$. However these agents are not absolutely without adverse effects.

Pneumocystis jirovecii, is an atypical fungus that transmits via airborne route in respiratory droplets. This agent is in dormitory state in immunocompetent hosts, and can transmit to others within sufficiently close distance [6]. It remains asymptomatic as long as the host would be immunocompetent [7]. Once the host becomes immunocompromised, the organism becomes activated and proliferated [8].

This agent has several components in its cell wall. The main component is $\beta$-1,3-glucan $[9,10]$. The first interaction is between macrophage receptor dectin- 1 and $\beta-1,3$-glucan $[11,12]$. This interaction leads to activation of lung macrophages and then releasing of several pro-inflammatory cytokines such as Tumor necrosis factor $\alpha(\mathrm{TNF} \alpha)$, Interleukin 8 (IL-8) [13-15]. This pro-inflammatory state results in neutrophil and other inflammatory cells recruitment, which is correlated directly to the lung inflammation, acute respiratory failure $[11,16]$. Patients with malignancy or immunodeficiency who have impaired in innate immune system function, cannot clear the fungi and are predispose to Pneumocystis jirovecii pneumonia (PCP). Also TNF blockade results in lack of immune response to Pneumocystis [17]. In addition to innate immunity, lymphocytes especially $\mathrm{CD}^{+}$and $\mathrm{CD}^{+}$play a major role in host defense against pneumocystis jirovecii. TNF, IL-1 and IL-8 directly stimulate T lymphocyte recruitment and activation [18, 19].

There are several risk factors that predispose people to developing PCP. The main factor is impaired cell mediated immunity, which has the essential role in defending against fungi. When CD4 counts drop below the 200 cells/mm as in HIV patients, Pneumocystis prophylaxis warranted. Other causes of predisposition are solid organ transplant especially renal, heart and lung transplant recipients $[20,21]$. In patients with hematological or oncological malignancies who receive chemotherapeutic regimens and become neutropenic, Pneumocystis infection is likely. Also in patients receiving corticosteroid more than 15-25 mg Prednisolone or more than $4 \mathrm{mg}$ Dexamethasone daily for more than 4 weeks are at higher risk of developing infection [22, 23]. These amounts of corticosteroid use are seen in chemotherapeutic regimens especially in lymphoblastic leukemia or some chemotherapeutic regimens of lymphoma. Higher dose of corticosteroid (more than $60 \mathrm{mg}$ daily) or concomitant use of Cyclophosphamide would have an additional risk in developing Pneumocystis infection [24]. Alemtuzumab that causes T cell depletion predisposes to Pneumocystis infection. Other chemotherapeutic regimens such as FCR (Fludarabine, Cyclophosphamide, and Rituximab), ABVD (Adriamycin, Bleomycin, Vinblastine, and Dacarbazine), R-CHOP (Rituximab, Cyclophosphamide, Adriamycin, 
Vincristine, and Prednisolone) in 14 days, also input patients into higher risk of Pneumocystis infection [25-28]. The risk of developing Pneumocystis infection in oncological malignancies are much lower than in hematological ones, so routine prescription of Pneumocystis prophylaxis are not recommended. In patients with brain tumors especially who receive an alkylating agent, Temozolamide as in patients with high grade gliomas, concurrent use of radiation therapy and also corticosteroid may increase the risk of infection [29]. Finally in patients with solid tumor who receive more than $20 \mathrm{mg}$ Prednisolone or equivalents for more than four weeks are at increased risk for Pneumocystis infection, so routine prophylaxis is recommended [30]. Restoration of CD4 counts with highly active antiretroviral therapy (HAART) in HIV patients or passing of nadir phase in patients receiving chemotherapy, leads to decrease the risk of developing infection [31].

In hematologic malignancies, risk of developing PCP depends strongly on the underlying disorders. Patients with AML have much lower risk of Pneumocystis infection and up to now there is no recommendation for prophylactic use of antimicrobials for Pneumocystis in these patients. Due to limited information about the PCP incidence in AML patients, this review assess data on PCP incidence and outcome in AML.

\section{Method}

This review conforms to the "Preferred Reporting Items for Systematic Reviews and Meta-Analyses" (PRISMA) statement [32].

\section{Search strategy}

PubMed and Embase databases were searched to retrieve relevant English language articles. Keywords for search strategy were as follows: ("Pneumonia, Pneumocystis" OR Pneumocystis Pneumonia" OR "Pneumocystis jirovecii"), AND ("leukemia, myeloid, acute OR acute myeloid leukemia").

Screening process and study selection

There were no restrictions on the article's publication date, but only studies written in English were selected. Searchers were performed by one of the authors. All potentially English relevant articles retrieved from each database were screened. Two reviewers independently reviewed titles and abstracts and chose articles fitting selection criteria for full-text evaluation. Discrepancies were discussed with a third reviewer. Irrelevant articles were excluded. Based on inclusion and exclusion criteria and full-text assessment, eligible studies were identified. Then the content was analyzed, and data were extracted (Figure 1).

Criteria for considering studies for this review

There were no restrictions on the article's publication date. We evaluated all case reports, case series and observational studies that were about the prevalence of PCP in patients with AML. Studies with relevant information were included in the final analysis. Titles, abstracts, and full texts of the recorded studies were evaluated based on the inclusion and exclusion criteria. The inclusion criteria were as follows: 1) studies including clinical trials, observational studies, case reports, case series and case letters on PCP and AML. We select papers with no date restriction which evaluated incidence of PCP in AML patients, clinical manifestations, chemotherapy regimen, phase of treatment and also outcome; 2) availability to the full-text articles; 3) only English language articles. Studies with at least one of the following aspects were excluded: 1) studies with non-relevant information, 2) studies with language other than English, 3) review articles and letters, 4) duplicate studies and 5) articles available only in abstract form. Appropriate papers were selected after evaluating all studies based on the inclusion and exclusion criteria.

\section{Data extraction}

The following data were extracted from the research included: authors, year of study, study type, number of patients, age, gender, chemotherapy regimen, phase of treatment and patient outcome. To abstract the papers, two authors evaluated different parts of the articles to ensure the relevance of the subject to the research objectives. Results of data classification were reviewed by one of the authors. In terms of article inclusion, if any disagreement existed between the two reviewers, another member of our research team was contacted to resolve any discrepancies. 


\section{Results:}

As shown in Figure 1, a total of 356 studies were identified from databases (316 in PubMed and 40 in Embase) and after deleting duplicates, 333 articles were reviewed based on the title and abstract. Of these, 311 articles were excluded, and 22 entered the next step. Then, the studies were reviewed based on the full-text and inclusion and exclusion criteria, and finally, seven papers were included in the study. Studies, classified into four case reports, two retrospective and one prospective study including totally 41 cases. Systematic review of cases with PCP in patients with AML are summarized in table 1 and 2. Table 1 shows the characteristics of patients with AML and PCP and table 2 shows the chemotherapy regimen, phase of treatment and outcomes [2, 33-38].

\section{Discussion:}

Based on studies and guidelines, lymphoproliferative disorders, acute and chronic lymphocytic leukemia and multiple myeloma constitute the vast majority of PCP cases in hematologic patients and PCP prophylaxis recommend in them [39, 40]. Based on guidelines, PCP in acute myeloid leukemia and myelodysplastic syndromes are rare and sparse and routine prophylaxis in these settings are not recommended. The reason for this recommendation maybe that PCP as fungi interacts with lymphocytes and in these myeloid disorders, the main abnormality is in myeloid precursors [26, 41, 42]. Another explanation is that steroids are used in chemotherapeutic regimens in lymphoproliferative disorders, lymphocytic leukemia and also multiple myeloma and this agent beside anti CD20 antibodies have no role in induction or consolidation therapy in myeloid disorders. The guidelines up to now recommend prophylaxis for Cytarabine only in lymphoproliferative disorders [43]. Unlike adults, in pediatric AML, routine prophylaxis for PCP is recommended [44].

Some studies focuses on the PCP incidence in AML patients. In a study conducted in Taiwan in 2018, 291 patients with AML were analyzed. 14 patients had definitive PCP and 6 patients had possible PCP. They found that there was an incidence of $6.8 \%$ risk of developing PCP in AML and $2 \%$ risk for each cycle of chemotherapy they received and the authors concluded that, prophylaxis should be considered in AML [33]. In contrast, Antoine R et al. in 2015, illustrated about two studies conducted in 1990-1999 and 2014 that among 55 and 321 patients with PCP, only 8 and 9 patients had AML respectively. The authors concluded that the diagnosis of PCP should not be part of the initial diagnostic process or therapy in patients with AML and the prophylaxis is not recommended [34, 45]. Some sparse case reports infavor of acute myeloid leukemia and PCP are available. In 2000, the authors presented a 30 year-old man with AML treated with subcutaneous low dose cytarabine. After one week he had signs and symptoms of sweet syndrome and PCP, with bilateral lung infiltration [37]. Lemez et al. treated 51 patients with AML and 3 patients with Myelodysplastic syndromes with Daunorubicin and Cytarabine. They received TMP-SMX for prophylaxis of pneumocystis. Only one of the eighteen patients without TMP-SMX prophylaxis during the first course of chemotherapy developed PCP that was treated with intravenous TMP-SMX [46]. An agent named Gemtuzumab ozogamicin is an anti CD33 monoclonal antibody which was approved for treatment of CD33+ AML in induction and consolidation therapy. CD33 is expressed on monocytes, granulocytes and myeloid progenitors and CD33 targeted therapies lead to myeloid cells depletion [47]. In results of reported trials for administering Gemtuzumab in AML patients, there was no increase in risk of infection due to this agent $[47,48]$.

Cytarabine which is the backbone of chemotherapy in myeloid disorders has an association with increased risk of PCP. Also it has been shown that $\mathrm{T}$ lymphoblasts are more active as compared to myeloblasts in cellular transport of cytarabine and this suggests that this agent is not only myelotoxic but is also profoundly immunosuppressive. Despite recovery of neutropenia, lymphopenia due to prolonged effect of cytarabine may make the patient susceptible to PCP.

We should also focuses on the role of alveolar macrophages. Macrophages are the front line of the antimicrobial defenses in the lung alveolar cavities. The major function of this cell and one of their most important roles is to fight against organisms and clean them across the alveolar cavities. Among these agents, 
Pneumocystis spp.are more sensitive to its killing functions. The most important biological manifestation of these cells is their ability to regenerate in this space. This makes them permanently present in this structure, so called self-renewing system. Given that they have tissue establishment at the beginning of the embryonic stages called: embryonic prenatal development. Therefore, they are present in the lungs under any circumstances. It is one of the cases that despite performing chemotherapy and bone marrow suppression during the treatment of AML patients, no serious damage is done to the above function and capability. Especially in the case of monoclonal antibodies therapies that, despite their positive effect on myeloid suppression in the bone marrow, are not able to eliminate alveolar macrophages because they have very low expression of CD33 surface marker. It can be concluded that in this malignancy, and during treatment, there is minimal damage to the function of these cells in killing Pneumocystis spp. and the need for less prophylactic antibiotic. Further studies can help and determine the true risk of PCP in patients with AML.

\section{Conclusion:}

The main point is that there are several patients with PCP belonging to categories not generally recommended to be treated with prophylactic cotrimoxazole and one of these categories are AML patients. Data in literature are so limited and sparse in association of pneumocystis in patients with AML. It seems that affected patients appear to have a clear resistance to PCP infection despite chemotherapy and bone marrow suppression due to the preservation of a specific immunological milieu in the lung. In fact, may be due to immunological stability of the lungs that remains intact. Further studies can determine the risk of PCP in patients with AML.

Declaration of Conflicting Interests: The authors declared no potential conflicts of interest with respect to the research, authorship, and/or publication of this article.

Funding : The authors received no financial support for the research, author-ship, and/or publication of this article.

\section{References:}

1. Matsumura, Y., et al., Clinical characteristics of Pneumocystis pneumonia in non-HIV patients and prognostic factors including microbiological genotypes. BMC infectious diseases, 2011. 11 (1): p. 1-9.

2. Pagano, L., et al., Pneumocystis carinii pneumonia in patients with malignant haematological diseases: 10 years' experience of infection in GIMEMA centres. British journal of haematology, 2002. 117 (2): p. 379-386.

3. Caselli, D., et al., Single-day trimethoprim/sulfamethoxazole prophylaxis for Pneumocystis pneumonia in children with cancer. The Journal of pediatrics, 2014. 164 (2): p. 389-392. e1.

4. Bucaneve, G., et al., Levofloxacin to prevent bacterial infection in patients with cancer and neutropenia. New England Journal of Medicine, 2005. 353 (10): p. 977-987.

5. Neumann, S., et al., Primary prophylaxis of bacterial infections and Pneumocystis jirovecii pneumonia in patients with hematological malignancies and solid tumors. Annals of hematology, 2013. 92 (4): p. 433-442.

6. Gigliotti, F., A. Harmsen, and T. Wright, Characterization of transmission of Pneumocystis carinii f. sp. muris through immunocompetent BALB/c mice. Infection and Immunity, 2003.71 (7): p. 3852-3856.

7. Morris, A. and K.A. Norris, Colonization by Pneumocystis jirovecii and its role in disease. Clinical microbiology reviews, 2012. 25 (2): p. 297-317.

8. Vargas, S.L., et al., Search for primary infection by Pneumocystis carinii in a cohort of normal, healthy infants. Clinical Infectious Diseases, 2001. 32 (6): p. 855-861.

9. Douglas, C., Fungal $\beta$ (1, 3)-D-glucan synthesis. Sabouraudia, 2001. 39 (1): p. 55-66. 
10. Roth, A., et al., Light and electron microscopy study of carbohydrate antigens found in the electronlucent layer of Pneumocystis carinii cysts. Parasitology research, 1997. 83 (2): p. 177-184.

11. Thomas, C.F. and A.H. Limper, Current insights into the biology and pathogenesis of Pneumocystis pneumonia. Nature Reviews Microbiology, 2007. 5 (4): p. 298-308.

12. Ricks, D.M., et al., Dectin immunoadhesins and pneumocystis pneumonia. Infection and immunity, 2013.81 (9): p. 3451-3462.

13. Steele, C., et al., Alveolar macrophage-mediated killing of Pneumocystis carinii f. sp. muris involves molecular recognition by the dectin-1 $\beta$-glucan receptor. The Journal of experimental medicine, 2003. 198 (11): p. $1677-1688$.

14. Vassallo, R., J.E. Standing, and A.H. Limper, Isolated Pneumocystis carinii cell wall glucan provokes lower respiratory tract inflammatory responses. The Journal of Immunology, 2000. 164 (7): p. 3755-3763.

15. Vassallo, R., et al., Vitronectin and fibronectin function as glucan binding proteins augmenting macrophage responses to Pneumocystis carinii. American Journal of Respiratory Cell and Molecular Biology, 2001. 25 (2): p. 203-211.

16. Benfield, T.L., et al., Prognostic value of interleukin-8 in AIDS-associated Pneumocystis carinii pneumonia. American journal of respiratory and critical care medicine, 1995. 151 (4): p. 1058-1062.

17. Chen, W., E.A. Havell, and A.G. Harmsen, Importance of endogenous tumor necrosis factor alpha and gamma interferon in host resistance against Pneumocystis carinii infection. Infection and immunity, 1992. 60 (4): p. 1279-1284.

18. Phair, J., et al., The risk of Pneumocystis carinii pneumonia among men infected with human immunodeficiency virus type 1. New England journal of medicine, 1990.322 (3): p. 161-165.

19. Shellito, J., et al., A new model of Pneumocystis carinii infection in mice selectively depleted of helper T lymphocytes. The Journal of clinical investigation, 1990.85 (5): p. 1686-1693.

20. Avino, L.J., S.M. Naylor, and A.M. Roecker, Pneumocystis jirovecii pneumonia in the non-HIV-infected population. Annals of Pharmacotherapy, 2016. 50 (8): p. 673-679.

21. Iriart, X., et al., Risk factors of Pneumocystis pneumonia in solid organ recipients in the era of the common use of posttransplantation prophylaxis. American journal of transplantation, 2015. 15 (1): p. 190-199.

22. Taplitz, R.A., et al., Antimicrobial prophylaxis for adult patients with cancer-related immunosuppression: ASCO and IDSA clinical practice guideline update. Journal of Clinical Oncology, 2018. 36 (30): p. 30433054 .

23. Yale, S.H. and A.H. Limper. Pneumocystis carinii pneumonia in patients without acquired immunodeficiency syndrome: associated illnesses and prior corticosteroid therapy. in Mayo Clinic Proceedings. 1996. Elsevier.

24. Park, J.W., et al., Prophylactic effect of trimethoprim-sulfamethoxazole for pneumocystis pneumonia in patients with rheumatic diseases exposed to prolonged high-dose glucocorticoids. Annals of the rheumatic diseases, 2018. 77 (5): p. 644-649.

25. Huang, Y.-C., et al., Low absolute lymphocyte count and addition of rituximab confer high risk for interstitial pneumonia in patients with diffuse large B-cell lymphoma. Annals of hematology, 2011. 90 (10): p. $1145-1151$.

26. Cordonnier, C., et al., Fifth European Conference on Infections in Leukemia (ECIL-5), a joint venture of The European Group for Blood and Marrow Transplantation (EBMT), The European Organization for 
Research and Treatment of Cancer (EORTC), the Immunocompromised Host Society (ICHS) and The European LeukemiaNet (ELN). Pneumocystis jirovecii pneumonia: still a concern in patients with haematological malignancies and stem cell transplant recipients. J Antimicrob Chemother, 2016. 71 (9): p. 2379-2385.

27. Kalin, M., et al., Fatal pneumocystis jiroveci pneumonia in ABVD-treated Hodgkin lymphoma patients. Annals of hematology, 2010. 89 (5): p. 523-525.

28. Haeusler, G.M., et al., Late-onset P neumocystis jirovecii pneumonia post-fludarabine, cyclophosphamide and rituximab: implications for prophylaxis. European journal of haematology, 2013. 91 (2): p. 157-163.

29. De Vos, F.Y., et al., Pneumocystis jirovecii pneumonia prophylaxis during temozolomide treatment for high-grade gliomas. Critical reviews in oncology/hematology, 2013. 85 (3): p. 373-382.

30. Sepkowitz, K.A., Opportunistic infections in patients with and patients without acquired immunodeficiency syndrome. Clinical infectious diseases, 2002.34 (8): p. 1098-1107.

31. Kaplan, J.E., et al., Epidemiology of human immunodeficiency virus-associated opportunistic infections in the United States in the era of highly active antiretroviral therapy. Clinical infectious diseases, 2000.30 (Supplement_1): p. S5-S14.

32. Moher, D., et al., Preferred reporting items for systematic reviews and meta-analyses: the PRISMA statement. PLoS medicine, 2009. 6 (7): p. e1000097.

33. Chang, H., et al., Pneumocystis jirovecii pneumonia in patients with acute myeloid leukaemia. Internal medicine journal, 2018. 48 (1): p. 81-83.

34. Roux, A., et al., Pneumocystis jirovecii pneumonia in patients with or without AIDS, France. Emerging infectious diseases, 2014. 20 (9): p. 1490.

35. Otsuka, M., et al., Torsades de pointes complicating pentamidine therapy of Pneumocystis carinii pneumonia in acute myelogenous leukemia. Intern Med, 1997.36 (10): p. 705-8.

36. Ong, Y.L. and F.G. Jones, A cluster of suspected Pneumocystis carinii Pneumonia following intensive chemotherapy in a Belfast haematology unit. Ulster Med J, 1998.67 (2): p. 104-9.

37. Chowdhary, V., et al., Sweet's syndrome and Pneumocystis carinii pneumonia: two sequelae of low-dose cytosine arabinoside therapy in a patient with acute myeloid leukemia. European journal of haematology, 2000. 65 (1): p. $72-73$.

38. Theron, J., et al., A 21-year-old patient with acute myeloid leukemia and bilateral pulmonary infiltrates. Respiration, 2001. 68 (6): p. 649-57.

39. Cooley, L., et al., Consensus guidelines for diagnosis, prophylaxis and management of $\mathrm{P}$ neumocystis jirovecii pneumonia in patients with haematological and solid malignancies, 2014. Internal medicine journal, 2014. 44 (12b): p. 1350-1363.

40. Maertens, J., et al., 5th European Conference on Infections in Leukaemia (ECIL-5), a joint venture of the European Group for Blood and Marrow Transplantation (EBMT), the European Organisation for Research and Treatment of Cancer (EORTC), the Immunocompromised Host Society (ICHS) and the European LeukemiaNet (ELN). ECIL guidelines for preventing Pneumocystis jirovecii pneumonia in patients with haematological malignancies and stem cell transplant recipients. J Antimicrob Chemother, 2016. 71 (9): p. 2397-2404.

41. Toma, A., et al., Infections in myelodysplastic syndromes. Haematologica, 2012. 97 (10): p. 1459.

42. Roblot, F., et al., Risk factors analysis for Pneumocystis jiroveci pneumonia (PCP) in patients with haematological malignancies and pneumonia. Scandinavian journal of infectious diseases, 2004. 36 (11-12): p. $848-854$. 
43. Obeid, K.M., et al., Risk factors for Pneumocystis jirovecii pneumonia in patients with lymphoproliferative disorders. Clinical Lymphoma Myeloma and Leukemia, 2012. 12 (1): p. 66-69.

44. Arad-Cohen, N., J.M. Rowe, and Y. Shachor-Meyouhas, Pharmacological prophylaxis of infection in pediatric acute myeloid leukemia patients. Expert opinion on pharmacotherapy, 2020. 21 (2): p. 193-205.

45. Antoine, R., B. Caroline, and V. François, All Patients with Leukemia Are Not Equally at Risk of Contracting Pneumocystis jirovecii Pneumonia. The American journal of medicine, 2015. 128 (1): p. e9.

46. Lemez, P., et al., Successful prevention of Pneumocystis carinii infection with 1,920 mg of trimethoprimsulfamethoxazole daily in patients with malignant hematopoietic diseases. Vnitrni lekarstvi, 1995. 41 (10): p. $688-691$.

47. Drgona, L., et al., ESCMID Study Group for Infections in Compromised Hosts (ESGICH) Consensus Document on the safety of targeted and biological therapies: an infectious diseases perspective (Agents targeting lymphoid or myeloid cells surface antigens [II]: CD22, CD30, CD33, CD38, CD40, SLAMF-7 and CCR4). Clinical Microbiology and Infection, 2018. 24 : p. S83-S94.

48. Castaigne, S., et al., Effect of gemtuzumab ozogamicin on survival of adult patients with de-novo acute myeloid leukaemia (ALFA-0701): a randomised, open-label, phase 3 study. The Lancet, 2012. 379 (9825): p. 1508-1516.

Figure 1. Flow chart of study selection for inclusion in the systematic review.

Table 1 . Characteristic of included studies.

\begin{tabular}{llllll}
\hline Year & References & Age/ mean age & Gender & No. of patients & Type of study \\
\hline 2018 & $\begin{array}{l}\text { Chang et al. } \\
(33)\end{array}$ & 55.5 & $\begin{array}{l}14 \text { Male } 6 \\
\text { Female }\end{array}$ & $20 / 291$ & Retrospective \\
2002 & $\begin{array}{l}\text { Pagano et al. } \\
(2)\end{array}$ & - & - & $8 / 55$ & Retrospective \\
2014 & $\begin{array}{l}\text { Roux et al. } \\
(34)\end{array}$ & - & - & $9 / 544$ & Prospective \\
1997 & $\begin{array}{l}\text { Otsuka et al. } \\
(35)\end{array}$ & 33 & Female & 1 & Case report \\
1998 & $\begin{array}{l}\text { Ong et al. (36) } \\
\text { Chowdhary et } \\
\text { al. (37) }\end{array}$ & 28 & Male & 1 & Case report \\
2000 & $\begin{array}{l}\text { Theron et al. } \\
(38)\end{array}$ & 21 & Male & 1 & Case report \\
2001 & & Male & 1 & Case report \\
\hline
\end{tabular}

Table 2. Chemotherapy regimen, phase of treatment and outcomes.

\begin{tabular}{llll}
\hline Year & $\begin{array}{l}\text { No. of patients/ Phase } \\
\text { of treatment }\end{array}$ & $\begin{array}{l}\text { No of patients/ } \\
\text { Chemotherapy }\end{array}$ & Outcome \\
\hline 2018 & 11/ Complete remission & 10/ HDAC 3/ FLAG 2/ & 6/ Deceased 14/ Cure \\
& 4/ Refractory 3/ Relapse & D 1/ EMA 1/ C 1/ E, C & \\
2/ New diagnosis & $\begin{array}{l}\text { 1/7+3 1/ None } \\
\text { 2/ Induction, }\end{array}$ & Not determined. & Not determined. \\
Re-induction 1/ & & \\
Consolidation 1/ & & \\
& Maintenance 1/ Salvage & & Not determined. \\
& 9/ Consolidation & Not determined. &
\end{tabular}




\begin{tabular}{llll}
\hline Year & $\begin{array}{l}\text { No. of patients/ Phase } \\
\text { of treatment }\end{array}$ & $\begin{array}{l}\text { No of patients/ } \\
\text { Chemotherapy }\end{array}$ & Outcome \\
\hline 1997 & Complete remission & $\begin{array}{l}\text { Daunorubicin, } \\
\text { Cytarabine, }\end{array}$ & Cure \\
& 6-Mercaptopurine & Cure \\
1998 & Complete remission & EMA & Deceased \\
2000 & Complete remission & Cytarabine & Daunorubicin, \\
2001 & Post consolidation & Cytarabine, Etoposide & \\
\hline
\end{tabular}

C, cytarabine; D, daunomycin; HDAC, high dose cytarabine; FLAG, fludarabine, cytarabine and granulocytestimulating factor; $7+3$, idarubicin (3 days) + cytarabine (7 days); EMA, Mitoxantrone, Cytarabine Etoposide; E, etoposide.

\section{Hosted file}

Tables.docx available at https://authorea.com/users/425847/articles/530557-pneumocystispneumonia-and-acute-myeloid-leukemia-a-systematic-review

\section{Hosted file}

Figures.docx available at https://authorea.com/users/425847/articles/530557-pneumocystispneumonia-and-acute-myeloid-leukemia-a-systematic-review 